\title{
A Block Scaling FFT/IFFT Processor for WiMAX Applications
}

\author{
Yuan Chen ${ }^{\dagger}$, Yu-Wei Lin ${ }^{\ddagger}$, and Chen-Yi Lee ${ }^{\dagger}$ \\ ${ }^{\dagger}$ National Chiao Tung University, Hsinchu, Taiwan \\ ${ }^{*}$ MediaTek Inc., Hsinchu, Taiwan \\ Email: ychen@si2lab.org
}

\begin{abstract}
This paper presents a low-power design of a twostream MIMO FFT/IFFT processor for WiMAX applications. A novel block scaling method and a new ping-pong cache-memory architecture are proposed to reduce the power consumption and hardware cost. With these schemes, half the memory accesses and 64-Kbit memory can be saved. Furthermore, by proper scheduling of the two data streams, the proposed design achieves better hardware utilization and can process two 2048-point FFTs/IFFTs consecutively within 2052 cycles. A test chip of the proposed FFT/IFFT processor has been designed using UMC $0.13 \mu \mathrm{m} 1$ P8M process with a core area of $1332 \times 1590 \mu \mathrm{m}^{2}$. The SQNR performance of the 2048-point FFT/IFFT is over $48 \mathrm{~dB}$ for QPSK and 16/64-QAM modulations. Power dissipation of two 2048-point FFT computations is about $17.26 \mathrm{~mW}$ at 22.86 MHz which meets the maximum throughput rate of WiMAX applications.
\end{abstract}

\section{INTRODUCTION}

Multiple-input multiple-output orthogonal frequency division multiplexing (MIMO OFDM) is considered a key technology in high-throughput transmissions over wireless fading channels. The emerging WiMAX/IEEE 802.16 standard has employed this technology in its physical-layer specification to provide broadband wireless access services. In the specification, scalable channel bandwidths from 1.25 to $20 \mathrm{MHz}$ by adjusting FFT size (from 128 to 2048-point) are employed for different applications. Three modulation types (QPSK, 16/64-QAM) and four guard intervals modes (1/4, $1 / 8,1 / 16,1 / 32)$ are also supported to further increase the system scalability. A block diagram of a $2 \times 2$ MIMO transceiver for WiMAX applications is shown in Fig. 1. By processing two data streams with duplicated antennas and functional units, the peak data rate of the $2 \times 2$ MIMO transceiver can be two-folded compared to that of a single-input single-output (SISO) transceiver.

To support a MIMO transceiver for WiMAX applications, a variable-length FFT/IFFT processor capable of processing multiple data streams is required. Since $2 \times 2$ MIMO with time division duplex (TDD) mode is defined in the WiMAX Forum Release-1 system profiles [1], a two-stream 128/256/ 512/1024/2048-point FFT/IFFT processor is considered in this paper. Besides, while the power consumption is critical for portable systems, the FFT/IFFT processor for WiMAX applications should be power-efficient. There have been many researches on low-power FFT designs by employing the cached-memory architecture to reduce the memory accesses [2], [3]. However, the increase in wordlength [2] or idle cycles [3] still causes wastes in power consumption and hardware cost. To solve these problems, a novel block scaling method and a new ping-pong cache-memory architecture are exploited in our proposed FFT/IFFT processor. With these schemes, half the memory accesses and 64-Kbit memory (4 bits in wordlength) can be saved without inducing idle cycles. Moreover, by proper scheduling of the two data streams, the proposed FFT/IFFT processor avoids stalls of function units and thus achieves better hardware utilization. Two-stream 2048-point FFTs/IFFTs can be computed consecutively within 2052 processing cycles.

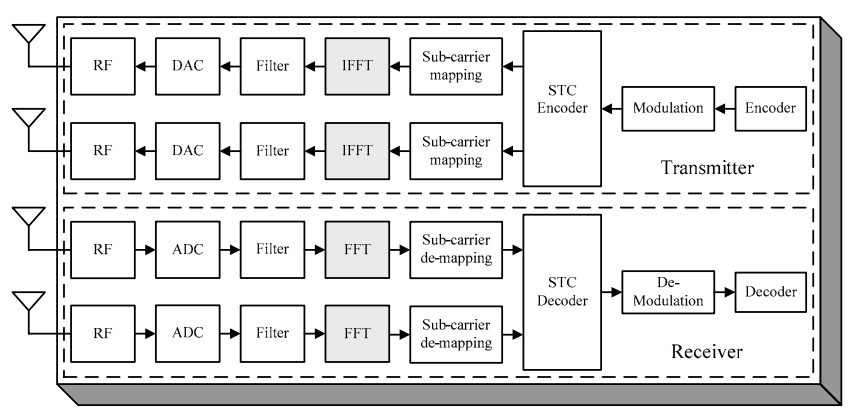

Fig. 1. Block diagram of a $2 \times 2$ MIMO transceiver for WiMAX applications.

\section{ALGORITHM}

The $N$-point discrete Fourier transform (DFT) of a complex input sequence $x(n)$ can be defined as:

$$
X(k)=\sum_{n=0}^{N-1} x(n) W_{N}^{k n} \quad k=0,1,2, \ldots N-1
$$

where $W_{N}^{k n}=e^{-j 2 \pi k n / N}$ is referred to the twiddle factor. To reduce the number of complex multiplications, radix- 8 algorithm is chosen to carry out the DFT [4]. Here we take the longest 2048-point DFT in the design as an example. Since 2048 is not a power of 8, we decompose the 2048-point DFT into three radix- 8 stages and a final radix-4 stage as shown in the following equation:

$$
\begin{aligned}
& X\left(k_{1}+8 k_{2}+64 k_{3}+512 k_{4}\right)=
\end{aligned}
$$

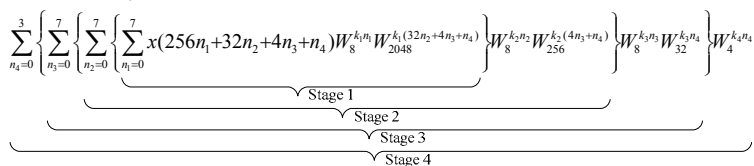

where $k_{1}, k_{2}, k_{3}=0,1,2, \ldots 7$ and $k_{4}=0,1,2,3$. Similarly, $128 / 256 /$ 512/1024-point DFT can also be decomposed to preceding 
radix- 8 stages and a final radix- $8 / 4 / 2$ stage depending on the DFT size. Although high-radix algorithm is effective in reducing the number of complex multiplications, its hardware is very complex if directly implemented. Thus we employ radix $-2^{3}$ and radix $-2^{2}$ [4] to replace radix- 8 and radix-4, respectively. A signal flow graph (SFG) of the 32-point radix $-2^{3} / 2^{2}$ FFT is shown in Fig. 2 as an example. We can find in this figure that a full 32-point FFT is completed by one radix $-2^{3}$ and multiplication stage and one radix $-2^{2}$ stage. With these steps, we can decompose the 2048-point FFT into three radix $-2^{3}$ and multiplication stages and a final radix $-2^{2}$ stage for further hardware implementations.

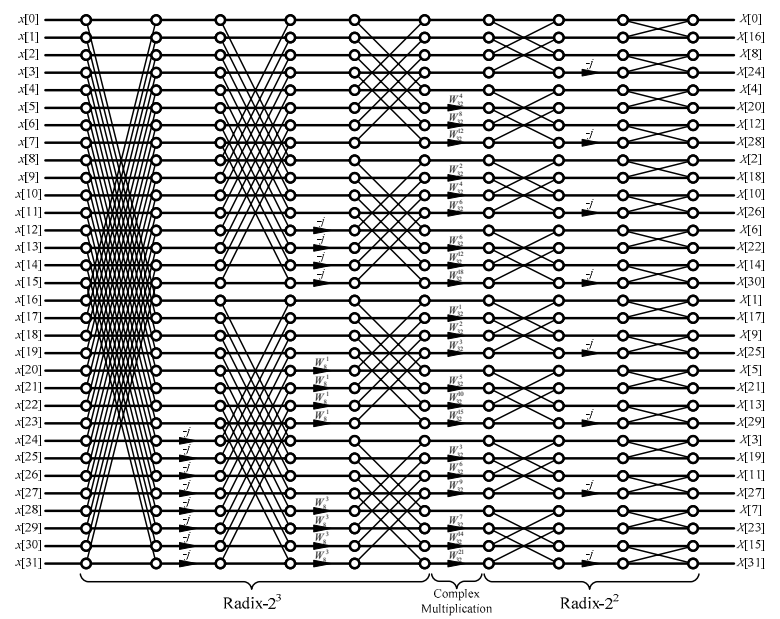

Fig. 2. SFG of a 32-point radix $-2^{3} / 2^{2} \mathrm{FFT}$.

\section{A. Block Scaling Method}

Block floating-point (BFP) [5] is an efficient way to reduce the wordlength by increasing the dynamic range compared to the fixed-point format. The behavior of BFP is similar to that of floating-point except a single exponent is used for a group of data. Although BFP is often adopted in memory-based FFT processors to save the hardware cost and power, it is not suited to cached-memory FFT processors because of the interleaved processing stages [5]. To solve this problem, a dynamic scaling FFT processor [3] is proposed by employing multiple exponents for cache-size blocks. While dynamic scaling approach has a satisfactory result in reducing wordlength, it still has two drawbacks. Since the exponent position can be determined only after all cached data are processed, some clock cycles are wasted. Also, the internal wordlength of both arithmetic units and cache needs to be extended to prevent overflows.

Thus we propose the block scaling method which eliminates the increased wordlength and idle cycles by a "detect and scale" approach. Each set of the output symbols will be scaled right away if an overflow is detected. At the same time, the resulting exponents are saved for data alignment in the next processing stage. Although this method can be realized by saving block exponents for all processing stages, it is hardware consuming. To work out this issue, we scale the final output of FFT to a predetermined exponent, and thus only 296 exponents are needed to be stored for the longest-length 2048-point FFTs. There are two main reasons why this fixed-exponent scheme is feasible. First, because the input symbols are gain-controlled and have specified modulation in OFDM systems, the maximum value of the final FFT output can be expected in advance. Second, in most dedicated OFDM transceiver designs, only fixed-point format is considered due to simpler hardware implementations. As the simulation result shows in Fig. 3, over four bits can be reduced in wordlength by the proposed method under the same signal-to-quantization-noise ratio (SQNR). We can also find that more than one fourth of the memory size (from 16 bits to 12 bits) can be saved at about $50 \mathrm{~dB}$ SQNR.

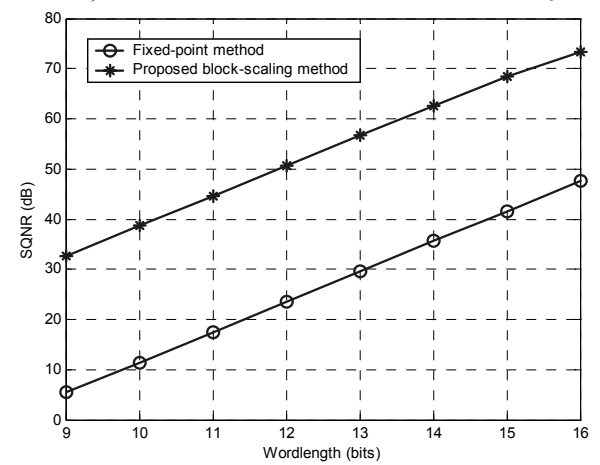

Fig. 3. SQNR performance of the proposed block scaling method.

\section{ARCHITECTURE}

Block diagram of the proposed FFT/IFFT processor is depicted in Fig. 4. It consists of four FFT/IFFT control units, a main memory unit, a processing engine (PE), and a 64-word cache. In this design, a novel block scaling method and a new ping-pong cache-memory architecture are proposed to reduce the power consumption and hardware cost. Besides, since FFT and IFFT have the same operations except for complexconjugated twiddle factors, we implement IFFT by simply taking conjugates of FFT input/output [6] as shown in Fig. 4. With these techniques and proper data scheduling, the proposed design can realize two 2048-point FFT/IFFT computations in 2052 clock cycles. Thus by taking the guard interval of WiMAX systems into account, the proposed FFT/IFFT processor does not need to operate in a multiple sampling frequency as the previous cached-memory FFT designs do [2], [3]. The modules of the proposed design will be described in more detail below.

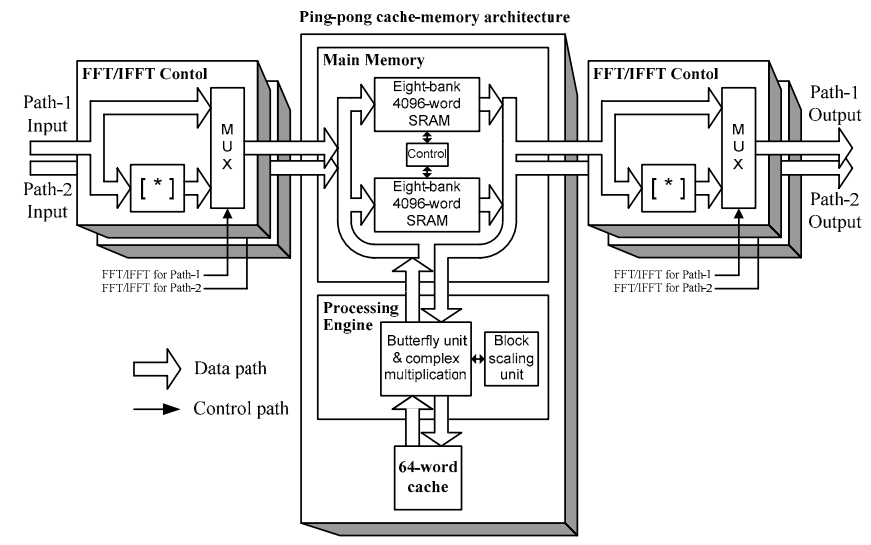

Fig. 4. Block diagram of the proposed two-stream FFT/IFFT processor. 


\section{A. Main Memory}

For memory-based FFT processors supporting consecutive $\mathrm{I} / \mathrm{O}$, multiple main memories are needed as computation and $\mathrm{I} / \mathrm{O}$ buffers [7]. To reduce the total memory size, the continuous flow (CF) memory architecture is proposed [7] where only two $\mathrm{N}$-word memories are required for $\mathrm{N}$-point FFT. Although CF FFT can reduce memory size by doing I/O operation concurrently in a single memory, it requires additional controls for memory addressing and butterfly units (BU). This is because the original CF FFT adopts radix-4 and radix-2 algorithms which have different bit-reverse orders. In our proposed design; however, CF memory architecture causes no problem since radix $-2^{3}$ and radix $-2^{2}$ algorithms have the same bit-reverse order as radix-2 algorithm [4]. As shown in Fig. 4, one 4096-word SRAM works as the I/O buffer while the other one works as the processing buffer, and vice versa. Each SRAM is further partitioned to eight banks to support eight accesses simultaneously for radix $-2^{3}$ algorithms.

\section{B. Ping-Pong Cache-Memory Architecture}

Cached-memory FFT [2], [3] is proposed for low power consumption by reducing the memory accesses. As shown in Fig. 5, data are first read from main memory and then sent to the cache. By proper data scheduling, PE can perform multiple-stage processing by accessing local cache instead of the main memory. Although cached-memory FFT can reduce memory accesses effectively, a concurrent read/write cache with complex control is required to increase the throughput. Thus we propose the ping-pong cache-memory architecture which uses a simple cache with single read/write operations. As illustrated in Fig. 6, data read from the main memory are processed by PE first and then written to the cache for future use. After the cache is full, data in the cache are read by PE and the computed results are stored back to the main memory. Since radix $-2^{3}$ algorithm is adopted in the proposed design, a 64-word cache is employed to support two-stage radix $-2^{3}$ processing. By using this scheme, half the memory accesses can be saved. Moreover, the ping-pong cache-memory has shorter latency compared to the cached-memory, which is beneficial in scheduling data streams.

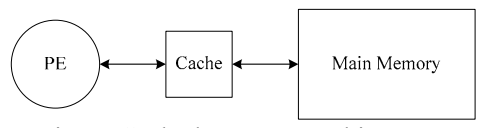

Fig. 5. Cached-memory architecture.

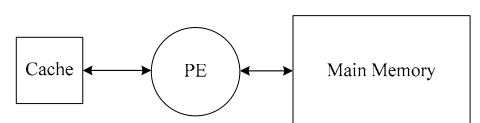

Fig. 6. Proposed ping-pong cache-memory architecture.

\section{C. $\quad$ Processing Engine (PE)}

The PE is designed to perform radix $-2^{3} / 2^{2} / 2$ butterfly operations and complex multiplications with proposed block scaling approach as shown in Fig. 7. Since variable-length FFT must be supported and the final stage can be radix $-2^{3}$, radix $-2^{2}$, or radix-2 as described earlier, a configurable radix $-2^{3} / 2^{2} / 2$ butterfly unit capable of processing one radix $-2^{3}$, two radix- $2^{2}$, or four radix-2 is adopted. We use 2048-point
FFT mode to describe the control of PE. At the fist processing stage, since the inputs have the same decimal point, data alignments are skipped. Input data are processed by radix $-2^{3}$ BU directly and then passed to the first overflow detection and scaling unit (ODSU1) in Fig. 7. If an overflow is detected, all eight inputs will be scaled and the corresponding shift in exponent is sent to the block scaling unit. Afterward, the output of ODSU1 is sent to the complex multipliers for twiddle factor multiplications. The outputs of the complex multipliers are passed to the second overflow detection and scaling unit (ODSU2) in Fig. 7 where the same operation of ODSU1 is performed. The second and third stages have similar control flows as stage 1 . For stage 4, after inputs are aligned in decimal point for processing, two radix $-2^{2}$ operations are performed. At this stage; however, only scaling is performed in ODSU1 since the final output is fixed-exponent in our proposed block scaling algorithm. Complex multiplications and ODSU2 are also skipped in this stage because no twiddle factor multiplication is required at final stage as shown previously in Fig. 2. The detailed control flow for all 128 2048 FFT modes is summarized in Table I.

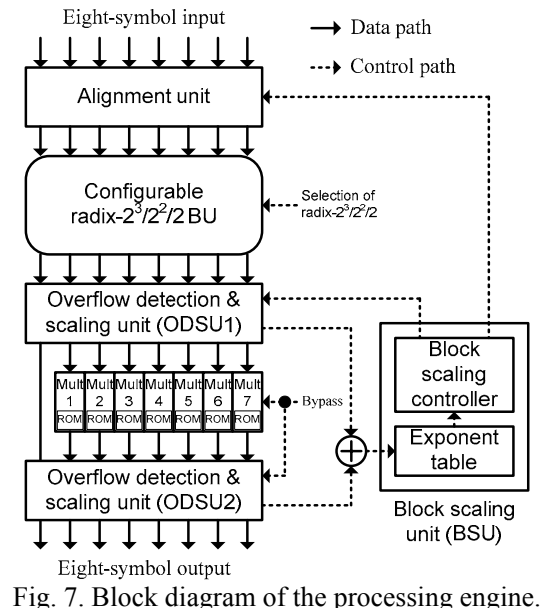

ABLE I. PE control for 128 2048-point FFT/IFFT.

\begin{tabular}{|l|l|l|l|}
\hline & \multicolumn{1}{|c|}{$\begin{array}{l}\text { First } \\
\text { Stage }\end{array}$} & $\begin{array}{l}\text { Intermediate } \\
\text { Stage(s) }\end{array}$ & \multicolumn{1}{c|}{$\begin{array}{l}\text { Final } \\
\text { Stage }\end{array}$} \\
\hline Alignment & Bypass & ON & ON \\
\hline $\begin{array}{l}\text { Configurable } \\
\text { BU }\end{array}$ & Radix-2 $^{3}$ & Radix-2 & $\begin{array}{l}\text { Radix-2 } \\
\text { Radix-2 } 2 \text { for 512 FFT } \\
\text { Radix-2 for 128/2048 FFT } \\
\text { Rad FFT }\end{array}$ \\
\hline ODSU1 & $\begin{array}{l}\text { Detection } \\
\text { \& Scaling }\end{array}$ & $\begin{array}{l}\text { Detection } \\
\text { \& Scaling }\end{array}$ & Scaling \\
\hline Multiplier & ON & ON & Bypass \\
\hline ODSU2 & $\begin{array}{l}\text { Detection } \\
\text { \& Scaling }\end{array}$ & $\begin{array}{l}\text { Detection } \\
\text { \& Scaling }\end{array}$ & Bypass \\
\hline $\begin{array}{l}\text { Block } \\
\text { scaling } \\
\text { unit }\end{array}$ & $\begin{array}{l}\text { Exponent } \\
\text { store }\end{array}$ & $\begin{array}{l}\text { Alignment } \\
\text { control \& } \\
\text { Exponent } \\
\text { store }\end{array}$ & $\begin{array}{l}\text { Alignment } \\
\text { control \& } \\
\text { ODSU1 } \\
\text { control }\end{array}$ \\
\hline
\end{tabular}

\section{CHIP IMPLEMENTATION}

A test chip of the proposed block scaling FFT/IFFT processor (2048-point mode) is implemented using UMC $0.13 \mu \mathrm{m} 1 \mathrm{P} 8 \mathrm{M}$ CMOS technology for verification. The core size is $1332 \times 1590 \mu \mathrm{m}^{2}$ as shown in Fig. 8. From post-layout prime power simulation, it is shown that the proposed 
FFT/IFFT consumes only $17.26 \mathrm{~mW}$ at $22.86 \mathrm{MHz}$ when performing two 2048-point FFT computations consecutively for WiMAX applications. The SQNR performance of the 2048-point FFT/IFFT has also been verified to exceed $48 \mathrm{~dB}$ for QPSK and 16/64-QAM signals. Thus the implementation loss of cascaded IFFT and FFT is only $0.1 \mathrm{~dB}$ with AWGN at $30 \mathrm{~dB}$ SNR which satisfies our design target for WiMAX applications. The detailed power profiling and chip summary are shown in Fig. 9.

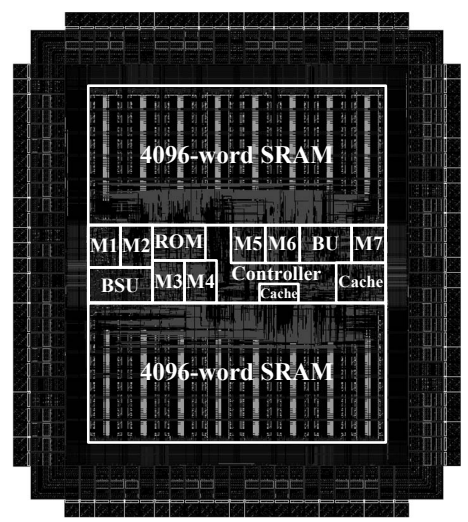

Fig. 8. Chip layout of the proposed FFT/IFFT Processor.
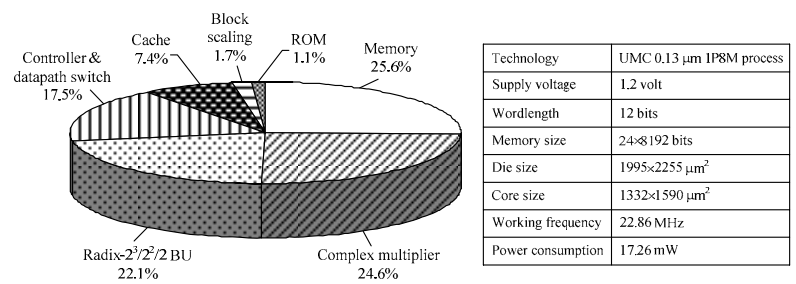

Fig. 9. Power profiling and chip summary of the proposed processor.

\section{COMPARISON}

For comparisons, we choose two FFT processor chips which can handle consecutive 2048-point FFT computations [8], [9]. Since these two chips can not support multiple data streams and only complete results for 1024-point FFT are listed, the comparisons of execution time and power are based on single-stream 1024-point FFT. Besides, to compare the FFT processor chips fabricated with different technologies, we adopt the normalized area and FFTs per energy [2] as our performance indices shown in eqs. (3) and (4). Note that eq. (4) has been adapted to take account of the voltage scaling.

$$
\begin{aligned}
& \text { Normalized Area }=\frac{\text { Area }}{(\text { Technology } / 0.13 \mu \mathrm{m})^{2}} \\
& \text { Normalized } \frac{\text { FFTs }}{\text { Energy }}=\frac{(\text { Technology } / 0.13 \mu \mathrm{m}) \times\left(V_{D D} / 1.2\right)^{2}}{\text { Power } \times \text { Execution Time } \times 10^{3}}
\end{aligned}
$$

The comparison results are summarized in TABLE II. We can find that the FFT processor [9] use a shorter wordlength of 12 bits since it only supports for 9-bit input. The processor [8] has employed the BFP approach and thus the wordlength is not increased. However, both designs [8], [9] do not employ a cache design to reduce the power of memory accesses. From this comparison, it is shown that our proposal has a satisfactory result in both normalized area and FFTs per energy, which justifies the feasibility of the proposed method.
TABLE II. Chip comparison of various 2048-point FFT Processors.

\begin{tabular}{|l|c|c|c|}
\hline & This Work & Zhong [8] & ${\text { Lin }[9]^{* 3}}^{*}$ \\
\hline Technology & $0.13 \mu \mathrm{m}$ & $0.25 \mu \mathrm{m}$ & $0.35 \mu \mathrm{m}$ \\
\hline $\begin{array}{l}\text { Supported FFT/ } \\
\text { IFFT (consecutive) }\end{array}$ & $\begin{array}{c}\text { Two 2048-point } \\
\text { FFTs/IFFTs }\end{array}$ & $\begin{array}{c}8 \sim 2048 \text {-point } \\
\text { FFT }\end{array}$ & $\begin{array}{c}512 ~ 2048 \text {-point } \\
\text { FFT }\end{array}$ \\
\hline Cache design & Yes & No & No \\
\hline Scaling/BFP design & Block scaling & BFP & No \\
\hline Input bit width & 12 bits & 16 bits & 9 bits \\
\hline Wordlength & 12 bits & 16 bits & 12 bits \\
\hline Core voltage & 1.2 volt & 2.5 volt & 3.3 volt \\
\hline Clock rate & $22.86 \mathrm{MHz}$ & $200 \mathrm{MHz}$ & $45.45 \mathrm{MHz}$ \\
\hline $\begin{array}{l}\text { Execution time } \\
\text { (1024-point) }\end{array}$ & $22.48 \mu \mathrm{ss}^{* 2}$ & $26.4 \mu \mathrm{s}$ & $45.06 \mu \mathrm{s}$ \\
\hline Power (1024-point) & $17.26 \mathrm{~mW}^{* 2}$ & $400 \mathrm{~mW}$ & $640 \mathrm{~mW}$ \\
\hline Core Area & $2.12 \mu \mathrm{m}^{2}$ & $11.42 \mu \mathrm{m}^{2}$ & $13.05 \mu \mathrm{m}^{2}$ \\
\hline $\begin{array}{l}\text { Normalized 1024- } \\
\text { Point FFTs/ Energy }\end{array}$ & $2577^{* 2}$ & 790 & 706 \\
\hline Normalized Area & 1.06 & 3.09 & 1.80 \\
\hline
\end{tabular}

\section{CONCLUSION}

A block scaling MIMO FFT/IFFT processor for WiMAX applications has been proposed in this paper. It can support two 2048-point FFT/IFFT computations simultaneously within 2052 clock cycles. Moreover, with a novel block scaling method and a new ping-pong cache-memory architecture, both power consumption and hardware cost can be greatly reduced. A test chip has been designed using UMC $0.13 \mu \mathrm{m} 1 \mathrm{P} 8 \mathrm{M}$ process. Simulation result has shown that the proposed FFT processor consumes only $17.26 \mathrm{~mW}$ at 22.86 MHz which meets the maximum throughput rate of WiMAX applications.

\section{ACKNOWLEDGMENT}

This work was supported by the National Science Council of Taiwan under Grant NSC94-2215-E-009-044 and by ICL/ITRI. under Grant 5352BA5115.

\section{REFERENCES}

[1] WiMAX Forum, Mobile WiMAX-Part I: A technical overview and performance evaluations, Feb. 21, 2006.

[2] B. M. Bass, "A low-power, high-performance, 1024-point FFT processor," IEEE J. Solid-State Circuits, vol. 34, pp. 380-387, Mar. 1999.

[3] Y.-W. Lin, H.-Y. Liu, and C.-Y. Lee, "A dynamic scaling FFT processor for DVB-T applications," IEEE J. Solid-State Circuits, vol. 39, pp. 2005-2013, Nov. 2004.

[4] He Shousheng and M. Torkelson, "Designing pipeline FFT processor for OFDM (de)modulation,” In Proc. Int. Symp. Signals, Systems, and Electronics, 29 Sept.-2 Oct. 1998, pp. 257-262.

[5] B. M. Baas, "An approach to low-power, high-performance, fast Fourier transform processor design," PhD Dissertation, Stanford University, Stanford, CA, 1999.

[6] K. Maharatna, E. Grass, and U. Jagdhold, "A 64-point Fourier transform chip for high-speed wireless LAN application using OFDM," IEEE J. Solid-State Circuits, vol. 39, pp. 484-493, Mar. 2003.

[7] B. G. Jo and M. H. Sunwoo, "New continuous-flow mixed radix (CFMR) FFT using novel in-place strategy," IEEE Trans. Circuits Syst., vol. 52, pp. 911-919, May. 2005.

[8] G. Zhong, F. Xu, and A. N. Willson Jr., "A power-scalable reconfigurable FFT/IFFT IC based on a multi-processor ring," IEEE J. Solid-State Circuits, vol. 41, pp. 483-495, Feb. 2006.

[9] Y.-T. Lin, P.-Y. Tsai, and T.-D. Chiueh, "Low-power variablelength fast Fourier transform processor," In Proc. Comput. Digit. Tech., vol. 152, No. 4, pp. 499-506, July 2005. 